\title{
Jean-Claude Quentel, Les fondements des sciences humaines, Érès, 2007
}

\section{Hubert Guyard}

\section{(2) OpenEdition}

Journals

Édition électronique

URL : https://journals.openedition.org/leportique/1563

DOI : 10.4000/leportique.1563

ISSN : $1777-5280$

\section{Éditeur}

Association "Les Amis du Portique"

Référence électronique

Hubert Guyard, « Jean-Claude Quentel, Les fondements des sciences humaines, Érès, 2007 », Le

Portique [En ligne], e-Portique, mis en ligne le 19 décembre 2007, consulté le 10 septembre 2022. URL : http://journals.openedition.org/leportique/1563 ; DOI : https://doi.org/10.4000/leportique.1563

Ce document a été généré automatiquement le 10 septembre 2022

Tous droits réservés 


\title{
Jean-Claude Quentel, Les fondements des sciences humaines, Érès, 2007
}

\author{
Hubert Guyard
}

1 Les sciences humaines se trouvent dans une phase extrêmement difficile : écartées dans le champ de la recherche par un mouvement cognitiviste qui se veut triomphant, elles sont à présent réfutées politiquement par nos gouvernants qui les accusent de ne pas répondre aux besoins du XXI ${ }^{\text {ème }}$ siècle. À l'université, elles subissent aujourd'hui une attaque sans précédent; on ferme ouvertement les équipes d'accueil et leurs masters. La psychanalyse, qui est à présent l'objet d'une chasse aux sorcières aux USA, résiste en France de plus en plus difficilement aux coups de butoirs qu'elle reçoit. On vise sa disparition comme discipline et comme champ de recherche. La sociologie, toujours suspecte de mettre en cause le pouvoir en place, se trouve dans une situation guère plus enviable. La linguistique, promue autrefois au rang de science pilote à l'intérieur des sciences humaines, rebaptisée depuis «sciences du langage ", a failli récemment disparaître de l'échiquier universitaire et on la somme de se ranger sous la bannière de la didactique des langues.

2 Sans nul doute, ces conditions politiques et sociales ne suffisent-elles pas à rendre compte de l'effacement actuel des sciences humaines. Celles-ci n'ont pas su tenir leurs promesses des années 1970-1980 et elles sont également responsables de la situation dans lesquelles elles se trouvent aujourd'hui. Le refus des modèles et le recours à une visée pragmatique a accéléré leur reflux, non seulement dans le champ de la recherche, mais aux yeux du grand public. Tous les éditeurs témoignent de la difficulté à vendre des ouvrages de sciences humaines, alors que la philosophie connaît parallèlement un engouement certain. Les disciplines des sciences humaines se sont par ailleurs repliées sur elles-mêmes et leurs travaux offrent la plupart du temps peu de lisibilité.

On peut se demander quel sera le temps que mettront les sciences humaines à traverser la forme de purgatoire qu'elles connaissent aujourd'hui. La question est cependant de savoir quels moyens elles vont se donner pour ne pas disparaître et affirmer leur 
importance en même temps que leur spécificité. Car il y a urgence. L'ouvrage de JeanClaude Quentel arrive à point nommé dans un tel climat. Il interroge en effet les fondements mêmes des sciences humaines et il propose par conséquent de faire retour sur ce qui les a nécessitées, c'est-à-dire sur ce qui fait qu'elles sont apparues à une époque précise de l'histoire en réponse à des questions que les approches scientifiques précédentes étaient incapables de résoudre. Il ne s'agit nullement dans ce travail de proposer une histoire des sciences humaines; il s'agit de mettre clairement à jour leur projet même et la problématique sur laquelle elles se sont élaborées. Leurs fondateurs ont en effet tous en commun d'avoir cerné, dans des champs différents, la spécificité des sciences humaines et d'avoir défini dans leurs lignes essentielles les particularités de l'humain.

4 Le livre de Jean-Claude Quentel, écrit dans un langage accessible, privilégiant les questions centrales, constitue non seulement un ouvrage destiné aux chercheurs et enseignants, mais aussi aux étudiants soucieux de comprendre, de façon synthétique, les enjeux épistémologiques de notre époque. Il se divise en trois grandes parties. La première interroge les sciences humaines du point de vue de leur prétention à la scientificité. Sont-elles scientifiques ou n'en ont-elles précisément que la prétention et qu'est-ce, dès lors, qu'une visée scientifique? Dans cette partie, l'auteur prend en compte toutes les objections qui sont faites aux sciences humaines, depuis leur origine jusqu'à aujourd'hui. Il les démonte en s'appuyant sur les arguments des fondateurs, arguments qui valent toujours aujourd'hui et qu'il importe précisément de rappeler aux détracteurs des sciences humaines, mais également à ceux qui ne voient pas l'intérêt de les promouvoir. La seconde partie traite de l'objet que les sciences humaines se donnent. Si science de l'humain il y a, la visée est la même que celle des autres sciences; en revanche l'objet diffère. Il n'est pas possible d'opérer comme si l'homme n'était pas un objet particulier, irréductible dans ce qui le particularise aux approches des sciences de la matière et de la vie. Quelle est exactement cette spécificité de l'homme ? C'est donc la réalité humaine, plus précisément sa rationalité, qui se trouve analysée dans cette partie. Cette rationalité apparaît toutefois multiforme: l'homme est «pluridimensionnel » et ceci rend compte des différentes approches de l'humain à l'intérieur des sciences humaines.

5 La dernière partie précise ce qu'il en est de cette réalité spécifique en mettant en évidence les caractéristiques de la rationalité humaine; ces caractéristiques valent en fait au-delà de la diversité des approches et traversent donc toutes les disciplines qui composent les sciences humaines. Une telle façon de questionner les sciences humaines à partir de leurs fondements se révèle nécessaire dans une période où les disciplines s'enferment sur elles-mêmes, en ignorant ce qui les rapproche les unes des autres. Il apparaît par conséquent possible de brosser un tableau d'ensemble des sciences humaines dans leur ressemblance en même temps que dans leur différence.

6 L'auteur fait remarquer en conclusion qu'un modèle répondant à ce souci de mise en ordre existe déjà : la « théorie de la médiation », élaborée par Jean Gagnepain, constitue un modèle anthropologique général qui situe de manière cohérente l'ensemble des « raisons » de l'humain. Encore insuffisamment connu, ce modèle pourrait constituer, par sa force heuristique, le fer de lance du renouveau des sciences humaines. Le philosophe Marcel Gauchet en a ainsi récemment souligné l'importance dans la préface à un dossier qui lui a été consacré dans la revue Le Débat (2006, n 140). Le modèle de 
Jean Gagnepain représente, dit-il, « l'une des percées majeures des sciences de l'homme au cours du dernier demi-siècle. Il n'est que temps de le découvrir ».

\section{AUTEURS}

\section{HUBERT GUYARD}

Professeur de Sciences du Langage à l'Université de Rennes 2, LIRL (EA 2241, Laboratoire d'Anthropologie et de Sociologie). 